\title{
METABOLIC SYNDROME AND CARDIOVASCULAR RISK IN FRAIL ELDERLY PEOPLE
}

\author{
Olga Vasovic ${ }^{1^{*}}$, Katarina Lalic ${ }^{2,3}$, Danijela Trifunovic ${ }^{2,4}$, Zvezdana Jemuovic ${ }^{3}$, Marina Nikolic-Djurovic ${ }^{2,3}$
}

${ }^{1}$ Institute for Gerontology and Palliative Care, Belgrade, Belgrade, Serbia, * e-mail: olga.vasovic@gmail.com

2 University of Belgrade, Faculty of Medicine, Belgrade, Serbia

${ }^{3}$ Clinic for Endocrinology, Diabetes and Metabolism, Clinical Center of Serbia, Belgrade, Serbia

${ }^{4}$ Clinic for Cardiology, Clinical Center of Serbia, Belgrade, Serbia

\section{Objectives}

Objective of this study was to examine the importance of metabolic syndrome (MetS) as a predictor of cardiovascular mortality in frail elderly people.

\section{Methods}

Participants of the study were 253 community dwelling elderly aged 65 to 99 years. It was a prospective study with 32 months follow-up period. Patients were divided into four main groups according to the presence of MetS and prior major cardiovascular event (MACE):

GROUP A- Patients without MetS and without MACE, the control group;

GROUP B- Patients with MetS, without MACE; GROUP C- Patients without MetS, with MACE; GROUP D- Patients with MetS and with MACE. We used Cox proportional hazards regression model for survival analysis. The results are presented as relative risk (RR) and 95\% confidence intervals $(95 \% \mathrm{Cl})$. Statistically significant differences were $\mathrm{p}<0.05$.

\section{Results}

The baseline mean age of participants was 82 years $(78.3 \%$ women); $53.8 \%$ had prior MACE. During the study 109 patients (43.1\%) died from cardiovascular cause of death. In our patients there was no statistically significant difference between four groups in functional ability to perform activity of daily living (ADL), smoking habits and systolic, diastolic and pulse pressure. Women were more represented in groups with MetS compared with groups without MetS. Control group was statistically older than group $D$ only. The best survival was in group B $(75.9 \%)$, and the worst in group C (43.8\%). Comparing to group $B$, participants in group $C$ have almost 3 times higher risk for cardiovascular mortality (RR 2.978; 95\% Cl: 1.605-5.523; $p=0.001$ ), whereas participants in group $D$ have 2.5 times higher cardiovascular mortality risk (RR 2.457; 95\% $\mathrm{Cl}: 1.273-4.744 ; p=0.01)$. Comparing to group A (the control group), only group $C$ have statistically higher mortality rate (RR $1.894 ; 95 \% \mathrm{Cl}: 1.145-$ 3.131; $p=0.01$ ).

\section{Conclusions}

In our study, presence of prior MACE, but not the presence of MetS raises risk for cardiovascular mortality in frail elderly people.

$17^{\text {th }}$ European Congress of Endocrinology,

16-20 May 2015, Dublin, Ireland

\section{Tables and Graphs}

Table 1. Selected baseline characteristics of participants

\begin{tabular}{|c|c|c|c|c|c|c|}
\hline $\begin{array}{c}\text { Baseline } \\
\text { characteristics }\end{array}$ & $\begin{array}{c}\text { GROUP A } \\
\mathrm{N}=63\end{array}$ & $\begin{array}{c}\text { GROUP B } \\
\mathrm{N}=54\end{array}$ & $\begin{array}{c}\text { GROUP C } \\
\mathrm{N}=80\end{array}$ & $\begin{array}{c}\text { GROUP D } \\
\qquad=56\end{array}$ & $\begin{array}{c}\text { All } \\
\text { participants } \\
\mathrm{N}=253\end{array}$ & $\begin{array}{c}\text { ANOVA } \\
p\end{array}$ \\
\hline $\begin{array}{l}\text { Age, years, } \\
\text { ( Mean } \pm \text { SD) }\end{array}$ & $83.6 \pm 5.3^{*}$ & $81.7 \pm 6.0$ & $80.9 \pm 7.9$ & $80.0 \pm 6.5^{*}$ & $81.6 \pm 6.7$ & 0.020 \\
\hline $\begin{array}{c}\text { Gender, female, } \\
(n, \%)\end{array}$ & $\begin{array}{c}47 \\
74.6 \%\end{array}$ & $\begin{array}{c}50 \\
92.6 \% *\end{array}$ & $\begin{array}{c}52 \\
65.0 \% *\end{array}$ & $\begin{array}{c}49 \\
87.5 \% *\end{array}$ & $\begin{array}{c}198 \\
78.3 \%\end{array}$ & $<0.001$ \\
\hline $\begin{array}{l}\text { Current smoker, } \\
\qquad(n, \%)\end{array}$ & $\begin{array}{c}6 \\
10.3 \%\end{array}$ & $\begin{array}{c}2 \\
4.2 \%\end{array}$ & $\begin{array}{c}4 \\
5.3 \%\end{array}$ & $\begin{array}{c}8 \\
15.4 \%\end{array}$ & $\begin{array}{c}20 \\
8.6 \%\end{array}$ & $>0.05$ \\
\hline $\begin{array}{c}A D L, \geq 2 \text { unable, } \\
(n, \%)\end{array}$ & $\begin{array}{c}8 \\
12.7 \%\end{array}$ & $\begin{array}{c}4 \\
7.4 \%\end{array}$ & $\begin{array}{c}13 \\
16.3 \%\end{array}$ & $\begin{array}{c}6 \\
10.7 \%\end{array}$ & $\begin{array}{c}31 \\
12.3 \%\end{array}$ & $>0.05$ \\
\hline $\begin{array}{c}\text { IADL, } \geq 3 \text { unable, } \\
(n, \%)\end{array}$ & $\begin{array}{c}32 \\
50.8 \%\end{array}$ & $\begin{array}{c}18 \\
33.3 \% *\end{array}$ & $\begin{array}{c}48 \\
60.0 \% *\end{array}$ & $\begin{array}{c}32 \\
57.1 \%\end{array}$ & $\begin{array}{c}130 \\
51.4 \%\end{array}$ & 0.017 \\
\hline Iypertensio & $\begin{array}{c}33 \\
52.4 \%\end{array}$ & $\begin{array}{c}41 \\
75.9 \% *\end{array}$ & $\begin{array}{c}38 \\
47.5 \% *\end{array}$ & $\begin{array}{c}34 \\
60.7 \%\end{array}$ & $\begin{array}{c}146 \\
57.7 \%\end{array}$ & 0.008 \\
\hline Diabet & $\begin{array}{c}19 \\
30.2 \%\end{array}$ & $\begin{array}{c}37 \\
68.5 \% *\end{array}$ & $\begin{array}{c}25 \\
31.3 \%\end{array}$ & $\begin{array}{c}39 \\
69.6 \% *\end{array}$ & $\begin{array}{c}120 \\
47.4 \%\end{array}$ & $<0.001$ \\
\hline
\end{tabular}

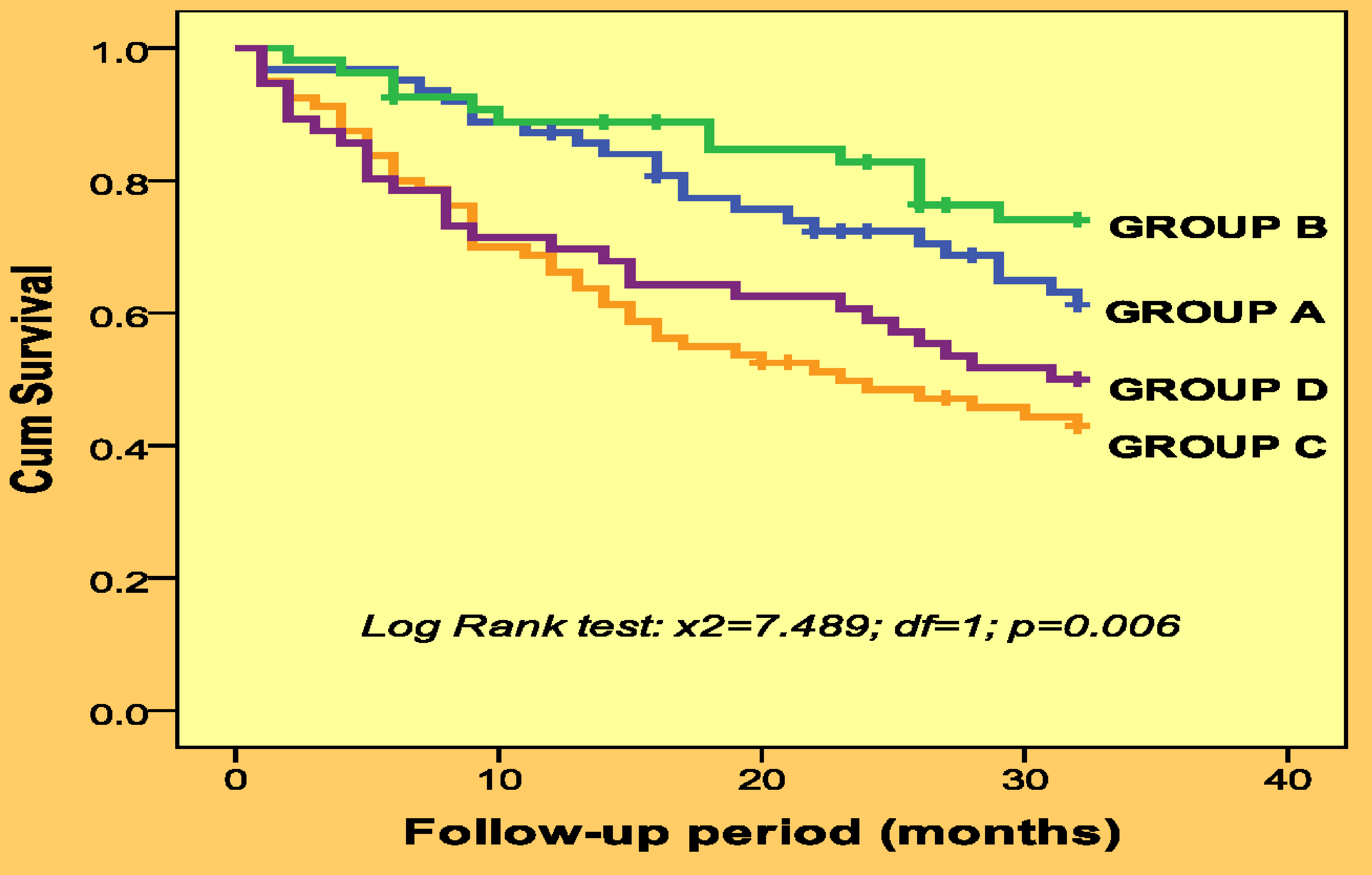

Figure 1. Kaplan-Meier curve for cardiovascular mortality

Table 2. Relative risk (RR) for cardiovascular mortality in groups comparing to group $A$

\begin{tabular}{|c|c|c|c|c|c|c|c|c|}
\hline GROUPS & $B$ & SE & Wald & df & Sig. $(p)$ & \multicolumn{1}{|c|}{ RR } & \multicolumn{2}{|c|}{$95.0 \%$ Cl } \\
\hline GROUP B & -0.453 & 0.347 & 1.701 & 1 & $>0.05$ & 0.636 & 0.322 & 1.256 \\
GROUP C & 0.638 & 0.257 & 6.193 & 1 & 0.013 & 1.894 & 1.145 & 3.131 \\
GROUP D & 0.446 & 0.281 & 2.515 & 1 & $>0.05$ & 1.563 & 0.900 & 2.713 \\
\hline
\end{tabular}

Table 3. Relative risk (RR) for cardiovascular mortality in groups comparing to group $B$

\begin{tabular}{|c|c|c|r|r|r|r|r|r|}
\hline GROUPS & $\boldsymbol{B}$ & SE & Wald & $\boldsymbol{d f}$ & Sig.(p) & $R R$ & \multicolumn{2}{|c|}{$95.0 \%$ Cl } \\
\hline GROUP A & 0.453 & 0.347 & 1.701 & 1 & $>0.05$ & 1.572 & 0.796 & 3.104 \\
GROUP D & 0.899 & 0.336 & 7.173 & 1 & 0.007 & 2.457 & 1.273 & 4.744 \\
GROUP C & 1.091 & 0.315 & 11.982 & 1 & 0.001 & 2.978 & 1.605 & 5.523 \\
\hline
\end{tabular}

